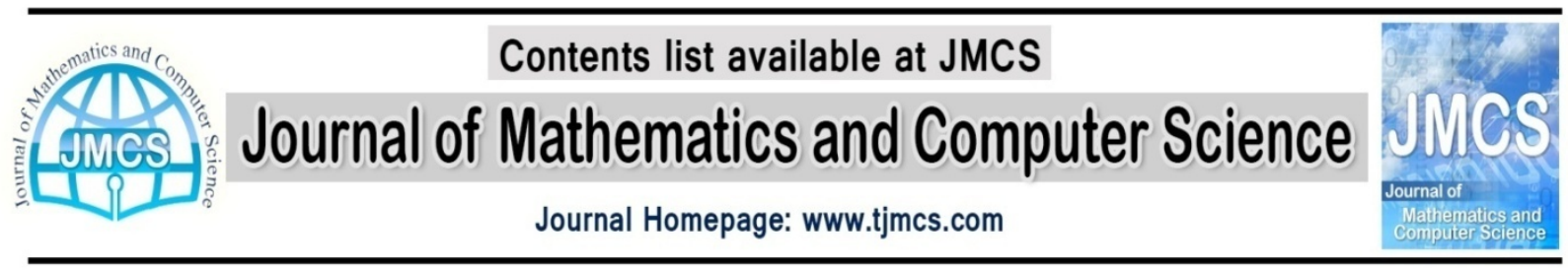

\title{
Common Fixed Point for Affine Self Maps Invariant Approximation in $\boldsymbol{p}$-normed Spaces
}

\author{
H. Shojaei, R. Mortezaei \\ Department of Mathematics, Payame- Noor- University, Tehran, Iran \\ hshojaei2000@yahoo.com
}

\begin{abstract}
Article history:
Received February 2013

Accepted March 20013

Available online April 2013
\end{abstract}

\begin{abstract}
Common fixed point results for new classes of non-commuting self-maps and non-expansive. Within the class of all self-maps $f$ and $T$ of a $w$-starshaped subset $M$ of $X$ where $f$ is affine or $w$-affine. We apply them to obtain several invariant approximation results which unify, extend, and complement well-known results.
\end{abstract}

Keywords: Best approximation; Common fixed point; affine self-maps; Invariant; $f$-nonexpansive; nonexpansive maps.

\section{Introduction and Preliminaries}

Recently, Latif [9] has obtained the following result on common fixed point in best approximations, which generalize and extend the recent study of AL- Thagafi [1], N. Hussain, M.A.Kutbi [5], etc. M. Abbas and J. Kyu kim[8] obtained common fixed point and invariant approximation results. AL. Thagafi and N.Shahzad[2, 3], common fixed point results are established for a new class of non-commuting selfmaps satisfying generalized $\mathrm{f}$-contraction or $\mathrm{f}$-non expansive type conditions and also extended several 
common fixed point and invariant approximation results, related to non- expansive self- maps, to the class

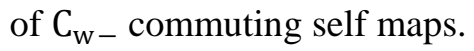

In this paper, we introduce two new classes of non- commuting self- maps. One class contains the weakly compatible self- maps as a proper subclass. Within the class of all self-maps $\mathrm{f}$ and $\mathrm{T}$ of a $\mathrm{w}$-star shaped subset $\mathrm{M}$ of $\mathrm{X}$ where $\mathrm{f}$ is $\mathrm{w}$-affine, the classes of commuting, $\mathrm{R}$-weakly commuting, and $\mathrm{C}_{\mathrm{w}}$ - commuting self maps, the classes of non expansive, $f$-non expansive, $f$-contraction and self maps are subclasses of the other class. For these new classes, we establish common fixed point results and obtain several invariant approximation results as applications. Our results unify, extend, and complement all the above mentioned results.

Definition 1.1 Let $X$ be a linear space. A $p$ - normed on $X$ is a real valued $\|\cdot\|_{p}$ on $X$ with $0<p \leq 1$ , satisfying the following conditions:

(i) $\|x\|_{p} \geq 0$ And $\|x\|_{p}=0$ if and only if $x=0$

(ii) $\|\beta x\|_{p}=|\beta|^{p}\|x\|_{p}$

(iii) $\|x+y\|_{p} \leq\|x\|_{p}+\|y\|_{p}$

For all $x, y \in X$ and all Scalars $\beta$, The pair $\left(X,\|\cdot\|_{p}\right)$ is called a $p$-normed space. It is a metric linear space with $d_{p}(x, y)=\|x-y\|_{p}$ for all $x, y \in X$, defining a translation invariant metric $d_{p}$ on $X$. If $p=1$ then we obtain the concept of a normed linear space. It is well- know that the topology of every Hausdorff locally bounded topological linear space is given by some $\mathrm{p}-$ normed $0 \leq p \leq 1$ (see [6]).

The space $l_{p}$ and $L_{p}[0,1], 0<p \leq 1$ are $p$-normed spaces. A $p$-normed space is not necessarily a Locally convex space, Recall that dual space $X^{*}$ separates points of $X$ if for each $0 \neq x \in X$, there exists $f \in X^{*}$ such that $f(x) \neq 0$. In this case the weak topology on $X$ is well-defined and is Hausdorff. Noticed that if $X$ is not locally convex Space, then $X^{*}$ need not separate the points of $X$. For example, if $X=$ $L_{p}[0,1], 0<p<1$ the power $p$ integerable function (or $X=S[0,1]$ ), the space of measurable functions, then $X^{*}=\{0\}$ (see [4, 10]). However, there are some non- locally convex spaces $X$, (such as the $p$-normed spaces $l_{p}, 0<p<1$ ) whose dual $X^{*}$ separates the points of $X$ [4]. In the sequel, we will assume that $X^{*}$ separates points of a $p$-normed space $X$ whenever weak topology is under consideration.

Definition 1.2 Let $M$ be a nonempty subset of a $p$-normed space $X$, and $f, T: X \rightarrow X$ are self- map of $M$.

a) A point $x \in X$ is called a fixed point of $f$ if $f x=x$

b) A point $x \in X$ is called a fixed point of $T$ if $T x=x$. We denote the set of fixed points of $f$ by $F(f)$ and the set of fixed points of $T$ by $F(T)$.

c) A point $x \in X$ is called a common fixed point of $f$ and $T$ if $f x=T x=x$. We denote the set of common fixed points of $f$ and $T$ by $F(f, T)$.

d) A point $x \in M$ is called a coincidence points of $f$ and $T$ if $f x=T x$. We denote the set of Coincidence points of $f$ and $T$ by $C(f, T)$. 
Definition 1.3: Let $M$ be subset of a $p$-normed space $X$.

a) For $w \in M$, the set $M$ is called $w$-star shaped if $k x+(1-k) w \in M$ for all $x \in M$ and all $k \in[0,1]$.

b) The set $M$ is called convex if $k x+(1-k) y \in M$ for all $x, y \in M$ and all $k \in[0,1]$.

\section{Definition 1.4:}

a) A self - mapping $f$ on a $p$-normed space $X$ is called affine If $M$ be convex and $f(k x+(1-k) y)=k f x+(1-k) f y$ for all $x, y \in M$ and all $k \in[0,1]$.

b) The self- map $f$ is $w$-affine if $M$ is $w$-star shaped and $f(k x+(1-k) w)=k f x+(1-k) f w$ for all $x \in M$ and all $k \in(0,1)$. Note that $f w=w$ whenever $f$ is a $w$-affine self map of a $w$ star shaped set $M$

Definition 1.5: Let $f, T$ be a self -maps of $\mathrm{M}$ in $p$-normed spaceX .

a) The self- maps $f$ and $T$ are called commuting if $f T x=T f x$ for all $x \in M$.

b) $R$-weakly commuting if $\|f T x-T f x\|_{p}<R\|f x-T x\|_{p}$ for all $x \in M$ and some $R>0$.

c) Weakly compatible if $f T x=T f x$ for all $x \in C(f, T)$.

d) $C_{w}$ - commuting if $M$ is $w$-starshaped with $w \in F(f)$ and $f T x=T f x$ for all $x \in C_{w}(f, T)=\cup\left\{C\left(f, T_{k}\right): \quad 0 \geq k \leq 1\right\}$ Where $T_{k} x=k T x+(1-k) w$

Definition 1.6: Let $T$ be a self- map of $p$-normed space $X$. The self- map $T$ is called $f-$ non expansive if $\|T x-T y\|_{p} \leq\|f x-f y\|_{p}$ for all $x, y \in M$ and non expansive if $\|T x-T y\|_{p} \leq$ $\|x-y\|_{p}$ for all $x, y \in M$ and $T$ a $f-$ contraction if $\|T x-T y\|_{p} \leq k\|f x-f y\|_{p}$ for all $x, y \in M$ and some $k \in(0,1)$

Definition 1.7: Let $X$ be a $p$-normed space. For a nonempty subset $S$ of $X$ and $x_{0} \in X$, define

$d_{p}\left(x_{0}, S\right)=i n f_{u \in S}\left\|x_{0}-u\right\|_{p}$

$P_{S}\left(x_{0}\right)=\left\{x \in S:\left\|x-x_{0}\right\|_{p}=d_{p}\left(x_{0}, S\right)\right\}$

The set $P_{S}\left(x_{0}\right)$ is called the set of best $S$-appropximants to $x_{0}$ and

$$
C_{S}^{f}\left(x_{0}\right)=\left\{x \in S: f x \in P_{S}\left(x_{0}\right)\right\}
$$

Note that $P_{S}\left(x_{0}\right)$ contain in $f\left(D_{S}^{f}\left(x_{0}\right)\right)$. Assume that $D_{S}^{f}\left(x_{0}\right)=P_{S}\left(x_{0}\right) \cap C_{S}^{f}\left(x_{0}\right)$ and $S_{x_{0}}=\left\{x \in S:\|x\|_{p} \leq 2\left\|x_{0}\right\|_{p}\right\}$.

Let $\mathcal{Q}_{0}$ denote the class of closed convex subsets of $X$ containing 0 . Note that $P_{S}\left(x_{0}\right) \subseteq S_{x_{0}} \in \mathcal{Q}_{0}$ Whenever $S \in \mathcal{Q}_{0}$. 
Theorem 1.8: Let $M$ be a subset of a $p$ - normed space $X, f$ and $T$ self maps of $M$, and $\overline{T(M)} \subseteq f(M)$. Suppose that $f$ and $T$ are weakly compatible, $T$ is a $f$-contraction, and $\overline{T(M)}$ is complete, then $F(f, T)$ is a singleton.

Proof: Let $x \in M$. As $T(M) \subseteq \overline{T(M)} \subseteq f(M)$, we can choose a sequence $\left\{x_{n}\right\}$ in $M$ such that $T x_{n-1}=f x_{n}$ for all $n \geq 1$ since $T$ is a $f$ - contraction, there exists $k \in(0,1)$ such that

$$
d_{p}\left(T x_{n+1}, T x_{n}\right) \leq k d_{p}\left(f x_{n+1}, f x_{n}\right)=k d_{p}\left(T x_{n}, T x_{n-1}\right)
$$

Therefore $\left\{T x_{n}\right\}$ is a Cauchy sequence in $T(M)$. It follows from the completeness of $\overline{T(M)}$ that $T x_{n} \rightarrow z \in \overline{T(M)} \subseteq f(M) \subseteq M$. Hence, $f x_{n} \rightarrow z$ where of $z=f u$ for some $u \in M$. Note that for all $n \geq 1$, we have;

$$
\begin{aligned}
d_{p}(z, T u) \leq & d_{p}\left(z, T x_{n}\right)+d_{p}\left(T x_{n}, T u\right) \\
& \leq d_{p}\left(z, T x_{n}\right)+k d_{p}\left(f x_{n}, f u\right) \\
& =d_{p}\left(z, T x_{n}\right)+k d_{p}\left(f x_{n}, z\right)
\end{aligned}
$$

Letting $n \rightarrow \infty$, we obtain $f u=z=T u$. Since $f$ and $T$ are weakly compatible on $M$, then $f z=$ $f T u=T f u=T z$. It follows that

$$
d_{p}(z, T z)=d_{p}(T u, T z) \leq k d_{p}(f u, f z)=k d_{p}(z, T z) .
$$

hence, $T z=z$ and so $f z=T z=z$. Clearly $F(f, T)$ is a singleton.

Theorem 1.8 contains theorem 2.1 of AL. Thagafi, N. shahzad [3] which established in metric space. We expressed in $p$ - normed space in this study.

Theorem 1.9: Let $M$ be a subset of a $p$-normed space $X, f$ and $T$ self- maps on $M$, and $\overline{T(M)} \subseteq$ $f(M)$. Suppose that $M$ is $w$-star shaped, $f$ and $T$ are $C_{w}$-commuting, $T$ is continuous and $f$-non expansive, $f$ is $w$-affine, and $\overline{T(M)}$ is compact, then $F(f, T)$ is nonempty.

Proof: Let $\left\{k_{n}\right\}$ be a sequence $n(0,1)$ such that $k_{n} \rightarrow 1$. For $n \geq 1$, define $T_{n}: M \rightarrow M$ by $T_{n} x=$ $k_{n} T x+\left(1-k_{n}\right) w$ for all $x \in M$. Since $M$ is $w$-star shaped, $\overline{T(M)} \subseteq f(M)$, and $f$ is $w$-affine, then $f w=w$ and $\overline{T_{n}(M)} \subseteq f(M)$ for all $n$. As $f$ and $T$ are $C_{w}$-commuting and $f$ is $w$-affine, then $f$ and $T_{n}$ are weakly compatible for all $n$. Since $T$ is $f$-nonexpansive, we have;

$\left\|T_{n} x-T_{n} y\right\|_{p}=\left(k_{n}\right)^{p}\|T x-T y\|_{p} \leq\left(k_{n}\right)^{p}\|f x-f y\|_{p}$, For all $x, y \in M$. Thus each $T_{n}$ is a $f$-contraction. As $\overline{T(M)}$ is compact, each $\overline{T_{n}(M)}$ is also compact. It follows from theorem 2.1 that $F\left(f, T_{n}\right)=\left\{x_{n}\right\}$ for some $x_{n} \in M$. Since $\left\{T x_{n}\right\}$ is a sequence in $\overline{T(M)}$, there exists a subsequence $\left\{T x_{n_{j}}\right\}$ of $\left\{T x_{n}\right\}$ and $z \in \overline{T(M)}$ such that $T x_{n_{j}} \rightarrow z$ so

$$
x_{n_{j}}=T_{n_{j}} x_{n_{j}}=k_{n_{j}} T x_{n_{j}}+\left(1-k_{n_{j}}\right) w \rightarrow z
$$


Now the continuity of $T$ implies that $T z=z$. Since $T(M) \subseteq f(M)$, there exists $u \in M$ such that $f u=$ $T z=z$. Moreover, for each $j$, we have;

$$
\left\|T x_{n_{j}}-T u\right\|_{p} \leq\left\|f x_{n_{j}}-f u\right\|_{p}=\left\|x_{n_{j}}-z\right\|_{p},
$$

Which, on letting $j \rightarrow \infty$.Implies that $f u=T z=z=T u$. So $C(f, T)$ is nonempty. Since $f$ and $T$ are also weakly compatible, we have

$$
f z=f T u=T f u=T z=z .
$$

Therefore, $F(f, T)$ is nonempty.

Corollary 1.10: Let $M$ be a subset of a $p$ - normed space, $T$ a self map of $M$, and $\overline{T(M)} \subseteq M$. Suppose that $M$ is $w$-starshaped, $T$ is nonexpansive, and $\overline{T(M)}$ is compact, then $F(T)$ is nonempty.

Definition 1.11: A map $T: M \rightarrow X$ is said to be semicompact if there is $\left\{x_{n}\right\}$ a sequence in $M$ such that $x_{n}-T x_{n} \rightarrow 0$ strongly, then $\left\{x_{n}\right\}$ has a subsequence $\left\{x_{n_{j}}\right\}$ in M such that $x_{n_{j}} \rightarrow z \in M$ strongly.

Note that if $\overline{T(M)}$ is compact, then $\overline{T(M)}$ is complete, bounded and $T$ is semicompact.

Definition 1.12: Let $f$ and $T$ be self maps of a subset $M$ of a $p$-normed space $X$. For every $x, y \in M$, define

$$
\begin{aligned}
& \varphi_{f, T}(x, y)=\max \left\{\|f x-f y\|_{p},\|f x-T x\|_{p},\|f y-T y\|_{p},\|f x-T y\|_{p},\|f y-T x\|_{p}\right\} \\
& \operatorname{dist}(f x, T y)=\inf \left\{\left\|f x-T_{k} y\right\|_{p}: 0 \leq k \leq 1\right\}, \text { and } \\
& \psi_{f, T}(x, y)=\max \left\{\|f x-f y\|_{p}, \operatorname{dist}(f x, T x), \operatorname{dist}(f y, T y), \operatorname{dist}(f x, T y), \operatorname{dist}(f y, T x)\right\} .
\end{aligned}
$$

If $f$ is the identity self maps of $M, \varphi_{f, T}(x, y)$ and $\psi_{f, T}(x, y)$ will be denoted by $\varphi_{T}(x, y)$ and $\psi_{T}(x, y)$, respectively, note that $\psi_{f, T}(x, y) \leq \varphi_{f, T_{k}}(x, y)$ and $\psi_{T}(x, y) \leq \varphi_{T_{k}}(x, y)$ for all $x, y \in M$ and all $k \in[0.1]$.

Theorem 1.13: Let $M$ be a subset of a $p$-normed space $X, f$ and $T$ self maps of $M, \overline{T(M)} \subseteq f(M)$, and $\overline{T(M)}$ complete. Suppose that $f$ and $T$ are occasionally weakly compatible and $d_{p}(T x, T y) \leq(k)^{p} \varphi_{f, T}(x, y)$ for all $x, y \in M$ and some $k \in[0,1)$ then $F(f, T)$ is a singleton.

The proof above theorem by AL-thagafi said in metric space [2]. The theorems follows extends from each of theorems $(2.3,2.4)$ of AL-thagafi and shahzad[2].

Theorem 1.14: Let $M$ be a $w$-starshaped subset of a $p$-normed space , $f$ and $T$ self maps of $M$, $\overline{T(M)} \subseteq f(M)=M$, and $\overline{T(M)}$ complete. Suppose that $T(M)$ is bounded, $T$ is semi compact, $f$ and $T$ are continuous and ultra occasionally weakly compatible, and $\|T x-T y\|_{p} \leq \psi_{f, T}(x, y)$ for all $x, y \in M$, Then $F(f, T)$ is nonempty. 
Proof: Let $\left\{k_{n}\right\}$ be a sequence in $(0,1)$ such that $k_{n} \rightarrow 1$ for $n \geq 1$, define $T_{n}: M \rightarrow M$ by $T_{n}=k_{n} T x+$ $\left(1-k_{n}\right) w$ for all $x \in M$. Since $M$ is $w$-starshaped, $\overline{T(M)} \subseteq M=f(M)$, and $\overline{T(M)}$ is complete, then each $\overline{T_{n}(M)}$ is complete and satisfies $\overline{T_{n}(M)} \subseteq M=f(M)$ moreover,

$$
\left\|T_{n} x-T_{n} y\right\|_{p} \leq\left(k_{n}\right)^{p}\|T x-T y\|_{p} \leq\left(k_{n}\right)^{p} \psi_{f, T}(x, y) \leq\left(k_{n}\right)^{p} \varphi_{T_{n}, f}(x, y)
$$

For all $x, y \in M$. Since $f$ and $T$ are ultra occasionally weakly compatible, then $f$ and $T_{n}$ are occasionally weakly compatible for all $n$. It follows from theorem 2.6 that $F\left(f, T_{n}\right)=\left\{x_{n}\right\}$ for some $x_{n} \in M$ since $\left\{x_{n}\right\}$ is bounded, $k_{n} \rightarrow 1$, and

$$
\left\|x_{n}-T x_{n}\right\|_{p}=\left\|f x_{n}-T x_{n}\right\|_{p} \leq\left(k_{n}^{-1}-1\right)^{p}\left(\|w\|_{p}+\left\|x_{n}\right\|_{p}\right)
$$

For all $n$, then $x_{n}-T x_{n} \rightarrow 0$ strongly, it follows from the semi compactness of $T$ that $\left\{x_{n}\right\}$ has a subsequence $\left\{x_{n_{j}}\right\}$ such that $x_{n_{j}} \rightarrow z \in M$ strongly. Since $T x_{n_{j}}=k_{n_{j}}{ }^{-1} x_{n_{j}}+\left(1-{k_{n_{j}}}^{-1}\right) w$, then $T x_{n_{j}} \rightarrow z$ strongly, by the continuity of both $f$ and $T$, we have $f z=T z=z$. therefore, $F(f, T)$ is nonempty.

\section{Main results}

Theorem 2.1: Let $X$ be a $p$-normed space, $f$ and $T$ self maps of $X$ with $x_{0} \in F(f, T)$, and $S \subseteq X$ with $T(\delta S \cap S) \subseteq S$. Suppose that $P_{S}\left(x_{0}\right)$ is closed and $w$-star shaped, $f$ and $T$ are $C_{w}$-commuting on $P_{S}\left(x_{0}\right), f\left(P_{S}\left(x_{0}\right)\right)=P_{S}\left(x_{0}\right), f$ is $w$-affine on $P_{S}\left(x_{0}\right), T$ is continuous on $P_{S}\left(x_{0}\right)$ and $f$-non expansive on $P_{S}\left(x_{0}\right) \cup\left\{x_{0}\right\}$, and $\overline{T\left(P_{S}\left(x_{0}\right)\right)}$ is compact, then $P_{S}\left(x_{0}\right) \cap F(I, T)$ is nonempty.

Proof: Let $x \in P_{S}\left(x_{0}\right)$. Then $\left\|k x+(1-k) x_{0}-x_{0}\right\|_{p}=(k)^{p}\left\|x-x_{0}\right\|_{p}<d_{p}\left(x_{0}, S\right)$ for all $k \in(0,1)$. Thus $\left\{k x+(1-k) x_{0}: k \in(0,1)\right\} \cap S=\emptyset$ and so $x \in \delta S \cap S$. Since $T(\delta S \cap S) \subseteq S$, it follow that $T x \in S$. Since $f x \in P_{S}\left(x_{0}\right)$ and $T$ is $f$-nonexpansive on $P_{S}\left(x_{0}\right) \cup\left\{x_{0}\right\}$, We have

$$
\left\|T x-x_{0}\right\|_{p}=\left\|T x-T x_{0}\right\|_{p} \leq\left\|f x-f x_{0}\right\|_{p}=\left\|f x-x_{0}\right\|_{p}=d_{p}\left(x_{0}, S\right)
$$

And hence $T x \in P_{S}\left(x_{0}\right)$, Therefore $\overline{T\left(P_{S}\left(x_{0}\right)\right)} \subseteq f\left(P_{S}\left(x_{0}\right)\right)=P_{S}\left(x_{0}\right)$. Now the result follows from theorem 1.9 with $M=P_{S}\left(x_{0}\right)$.

Theorem2.2: Let $X$ be a $p$-normed space, $f$ and $T$ self-maps of $X$ with $x_{0} \in F(f, T)$ and $S \in \mathcal{Q}_{0}$ with $T\left(S_{x_{0}}\right) \subseteq f(S) \subseteq S$. Suppose that $\left\|f x-x_{0}\right\|_{p}=\left\|x-x_{0}\right\|_{p}$ for all $x \in S$, $T$ is $f$-nonexpansive on $S_{x_{0}} \cup\left\{x_{0}\right\}$, and $\overline{f\left(S_{x_{0}}\right)}$ is compact.. Then $P_{S}\left(x_{0}\right)$ is nonempty, closed, and convex and $T\left(P_{S}\left(x_{0}\right)\right) \subseteq$ $f\left(P_{S}\left(x_{0}\right)\right) \subseteq P_{S}\left(x_{0}\right)$. If, in addition, $f$ is nonexpansive on $P_{S}\left(x_{0}\right)$, then $P_{S}\left(x_{0}\right) \cap F(f)$ and $P_{S}\left(x_{0}\right) \cap F(T)$ are nonempty. If, for some $w \in P_{S}\left(x_{0}\right), f$ is $w$-affine, $f$ and $T$ are $C_{w}$-commuting on $P_{S}\left(x_{0}\right)$, and $\overline{T\left(P_{S}\left(x_{0}\right)\right)} \subseteq f\left(P_{S}\left(x_{0}\right)\right)$, then $P_{S}\left(x_{0}\right) \cap F(f, T)$ is nonempty.

Proof: We may assume that $x_{0} \notin S$. If $x \in S \backslash S_{x_{0}}$, then $\|x\|_{p}>2\left\|x_{0}\right\|_{p}$ and, so

$$
\left\|x-x_{0}\right\|_{p} \geq\|x\|_{p}-\left\|x_{0}\right\|_{p}>\|x\|_{p} \geq d_{p}\left(x_{0}, S\right) .
$$


Thus $\alpha=d_{p}\left(x_{0}, S_{x_{0}}\right)=d_{p}\left(x_{0}, S\right)$. Suppose $\overline{f\left(S_{x_{0}}\right)}$ is compact. Since the norm is continuous, there exists $z \in \overline{f\left(S_{x_{0}}\right)}$ such that $\beta=d_{p}\left(x_{0}, \overline{f\left(S_{x_{0}}\right)}\right)=\left\|z-x_{0}\right\|_{p}$. Hence

$$
\alpha \leq \beta \leq d_{p}\left(x_{0}, f\left(S_{x_{0}}\right)\right) \leq\left\|f y-x_{0}\right\|_{p}=\left\|y-x_{0}\right\|_{p}
$$

For all $y \in S_{x_{0}}$. This show $\alpha=\beta$. Thus, $P_{S}\left(x_{0}\right)$ is nonempty, closed, and convex and $f\left(P_{S}\left(x_{0}\right)\right) \subseteq$ $P_{S}\left(x_{0}\right)$. To show that $T\left(P_{S}\left(x_{0}\right)\right) \subseteq f\left(P_{S}\left(x_{0}\right)\right)$, let $y \in T\left(P_{S}\left(x_{0}\right)\right)$. Since $T\left(P_{S}\left(x_{0}\right)\right) \subseteq T\left(S_{x_{0}}\right) \subseteq f(S)$, there exists $z \in P_{S}\left(x_{0}\right)$ and $w \in S$ such that $y=T z=f w$. Then the $f$-nonexpansiveness of $T$ on $S_{x_{0}} \cup\left\{x_{0}\right\}$ implies

$$
\left\|w-x_{0}\right\|_{p}=\left\|f w-x_{0}\right\|_{p}=\left\|T z-x_{0}\right\|_{p} \leq\left\|f z-x_{0}\right\|_{p}=\left\|z-x_{0}\right\|_{p}=d_{p}\left(x_{0}, S\right) .
$$

Thus, $w \in P_{S}\left(x_{o}\right)$ and $y \in f\left(P_{S}\left(x_{0}\right)\right)$. Hence, $T\left(P_{S}\left(x_{0}\right)\right) \subseteq f\left(P_{S}\left(x_{0}\right)\right) \subseteq P_{S}\left(x_{0}\right)$. Now, suppose that $f$ is nonexpansive on $P_{S}\left(x_{0}\right)$. Then the $f$-nonexpansive ness of $T$ on $S_{x_{0}} \cup\left\{x_{0}\right\}$ implies that $T$ is nonexpansive on $P_{S}\left(x_{0}\right)$. Since $\overline{f\left(S_{x_{0}}\right)}$ is compact, then $\overline{f\left(P_{S}\left(x_{0}\right)\right)}$ and $\overline{T\left(P_{S}\left(x_{0}\right)\right)}$ are compact. Moreover, $\overline{f\left(P_{S}\left(x_{0}\right)\right)} \subseteq P_{S}\left(x_{o}\right)$ and $\overline{T\left(P_{S}\left(x_{0}\right)\right)} \subseteq P_{S}\left(x_{o}\right)$. It follows from corollary 1.10 that $P_{S}\left(x_{o}\right) \cap$ $F(f)$ and $P_{S}\left(x_{o}\right) \cap F(T)$ are nonempty. The last part follows from theorem 2.2 with $=P_{S}\left(x_{o}\right)$.

Theorem 2.3: Let $M$ be a $w$-starshaped subset of a $p$-normed space, $f$ and $T$ self maps of $M$, $\overline{T(M)} \subseteq f(M)$, and $\overline{T(M)}$ complete. Suppose that $f$ is $w$-affine, $T(M)$ is bound, $T$ is semicompact, $f$ and $T$ are continuous and ultra occasionally weakly compatible, and $\|T x-T y\|_{p} \leq \psi_{f, T}(x, y)$ for all $x, y \in M$, Then $F(f, T)$ is nonempty.

Proof: Let $\left\{k_{n}\right\}$ and $\left\{T_{n}\right\}$ be as in the proof of theorem 1.14. Since $M$ is $w$-starshaped, $\overline{T(M)} \subseteq f(M)$, $\overline{T(M)}$ is complete, and $f$ is $w$-affine, Then each $\overline{T_{n}(M)}$ is complete and satisfies $\overline{T_{n}(M)} \subseteq f(M)$. Moreover;

$$
\left\|T_{n} x-T_{n} y\right\|_{p}=\left(k_{n}\right)^{p}\|T x-T y\|_{p} \leq\left(k_{n}\right)^{p} \psi_{f, T}(x, y) \leq\left(k_{n}\right)^{p} \varphi_{f, T_{n}}(x, y)
$$

For all $x, y \in M$. Since $f$ and $T$ are ultra occasionally weakly compatible, then $f$ and $T_{n}$ are occasionally weakly compatible for all $\mathrm{n}$. It follows from theorem 1.13 that $\mathrm{F}\left(f, T_{n}\right)=\left\{x_{n}\right\}$ for some $x_{n} \in M$, Since $\left\{x_{n}\right\}$ is bounded, $k_{n} \rightarrow 1$, and

$$
\left\|x_{n}-T x_{n}\right\|_{p}=\left\|I x_{n}-T x_{n}\right\|_{p} \leq\left(k_{n}^{-1}-1\right)^{p}\left(\|w\|_{p}+\left\|x_{n}\right\|_{p}\right)
$$

For all $n$, then $x_{n}-T x_{n} \rightarrow 0$ strongly, it follows from the semi compactness of $T$ that $\left\{x_{n}\right\}$ has a subsequence $\left\{x_{n_{j}}\right\}$ such that $x_{n_{j}} \rightarrow z \in M$ strongly. Since $T x_{n_{j}}=k_{n_{j}}{ }^{-1} x_{n_{j}}+\left(1-k_{n_{j}}{ }^{-1}\right) w$

Then $T x_{n_{j}} \rightarrow z$ strongly, By the continuity of both $f$ and $T$, we have $f z=T z=z$ therefore, $F(f, T)$ is nonempty. $\square$ 
Theorem 2.4: Let $X$ be a $p$-normed space, $f$ and $T$ self-maps of $X$ with $x_{0} \in X$, and $S \subseteq X$ with $T(\delta S \cap S) \subseteq S$. Suppose that ${C_{S}}^{f}\left(x_{0}\right)$ is closed and $w$-starshaped, $f\left(C_{S}{ }^{f}\left(x_{0}\right)\right)=C_{S}{ }^{f}\left(x_{0}\right), \overline{T\left(C_{S}{ }^{f}\left(x_{0}\right)\right)}$ is complete, and $T$ is semi compact on $C_{S}{ }^{f}\left(x_{0}\right), f$ and $T$ are continuous and ultra -occasionally weakly compatible on $C_{S}{ }^{f}\left(x_{0}\right)$, $\|T x-T y\|_{p} \leq \psi_{f, T}(x, y)$ for all $x, y \in C_{S}{ }^{f}\left(x_{0}\right)$,

$\left\|T x-x_{0}\right\|_{p} \leq\left\|f x-x_{0}\right\|_{p}$ for all $x \in C_{S}{ }^{f}\left(x_{0}\right)$, and $\left\|f x-x_{0}\right\|_{p}=\left\|x-x_{0}\right\|_{p}$ for all $x \in T\left(C_{S}{ }^{f}\left(x_{0}\right)\right)$, Then $P_{S}\left(x_{o}\right) \cap F(f, T)$ is nonempty.

Proof: Let $x \in{C_{S}}^{f}\left(x_{0}\right)$. As ${C_{S}}^{f}\left(x_{0}\right)=f\left(x_{0}\right) \subseteq P_{S}\left(x_{0}\right)$, then $x \in P_{S}\left(x_{0}\right)$. Therefore $\left\|k x+(1-k) x_{0}-x_{0}\right\|_{p}=(k)^{p}\left\|x-x_{0}\right\|_{p}<d_{p}\left(x_{0}, S\right)$ for all $k \in(0,1)$. Thus

$\left\{k x+(1-k) x_{0}: k \in(0,1)\right\} \cap S=\emptyset$ and, hence $x \in \delta S \cap S$. Since $\left\|T x-x_{0}\right\|_{p} \leq\left\|f x-x_{0}\right\|_{p}$ and $f x \in P_{S}\left(x_{0}\right)$, then $T x \in P_{S}\left(x_{0}\right)$. Since $\left\|f T x-x_{0}\right\|_{p}=\left\|T x-x_{0}\right\|_{p}$ and $T x \in T\left(C_{S}{ }^{f}\left(x_{0}\right)\right) \cap P_{S}\left(x_{0}\right)$,

Then $T x \in C_{S}{ }^{f}\left(x_{0}\right)$. Thus $\overline{T\left(C_{S}{ }^{f}\left(x_{0}\right)\right)} \subseteq f\left(C_{S}{ }^{f}\left(x_{0}\right)\right)=C_{S}{ }^{f}\left(x_{0}\right)$. Now the result follows from theorem 2.7 with $M=C_{S}{ }^{f}\left(x_{0}\right)$.

Theorem 2.5 Let $X$ be a $p$-normed space, $f$ and $T$ self-maps of $X$ with $x_{0} \in X$, and $S \subseteq X$ with $T(\delta S \cap$ $S) \subseteq S$. Suppose that $D_{S}{ }^{f}\left(x_{0}\right)$ is closed and $w$-starshaped, $f\left(D_{S}^{f}\left(x_{0}\right)\right)=D_{S}{ }^{f}\left(x_{0}\right), \overline{T\left(D_{S}{ }^{f}\left(x_{0}\right)\right)}$ is complete, and $T$ is semicompact on $D_{S}{ }^{f}\left(x_{0}\right), f$ and $T$ are continuous and ultra -occasionally weakly compatible on $D_{S}{ }^{f}\left(x_{0}\right),\|T x-T y\|_{p} \leq \psi_{f, T}(x, y)$ for all $x, y \in D_{S}{ }^{f}\left(x_{0}\right)$,

$\left\|T x-x_{0}\right\|_{p} \leq\left\|f x-x_{0}\right\|_{p}$ for all $x \in D_{S}{ }^{f}\left(x_{0}\right)$, and $\left\|f x-x_{0}\right\|_{p}=\left\|x-x_{0}\right\|_{p}$ for all $x \in T\left(D_{S}{ }^{f}\left(x_{0}\right)\right)$, Then $P_{S}\left(x_{o}\right) \cap F(f, T)$ is nonempty.

Proof: Let $x \in D_{S}{ }^{f}\left(x_{0}\right)$. As ${D_{S}}^{f}\left(x_{0}\right) \subseteq P_{S}\left(x_{0}\right)$, therefore $x \in P_{S}\left(x_{0}\right)$. Then; $\left\|k x+(1-k) x_{0}-x_{0}\right\|_{p}=(k)^{p}\left\|x-x_{0}\right\|_{p}<d_{p}\left(x_{0}, S\right)$ For all $k \in(0,1)$. Thus; $\left\{k x+(1-k) x_{0}: k \in(0,1)\right\} \cap S=\emptyset$ And, hence $x \in \delta S \cap S$.

Since $T(\delta S \cap S) \subseteq S$, then $T x \in S$. As $\left\|T x-x_{0}\right\|_{p} \leq\left\|f x-x_{0}\right\|_{p}$ and $f x \in P_{S}\left(x_{0}\right)$, we have $T x \in$ $P_{S}\left(x_{0}\right)$. Since $\left\|f T x-x_{0}\right\|_{p}=\left\|T x-x_{0}\right\|_{p}$ and $T x \in T\left(D_{S}{ }^{f}\left(x_{0}\right)\right) \cap P_{S}\left(x_{0}\right)$, Then $T x \in C_{S}{ }^{f}\left(x_{0}\right)$, thus $T x \in D_{S}^{f}\left(x_{0}\right)$, and hence $\overline{T\left(D_{S}{ }^{f}\left(x_{0}\right)\right)} \subseteq f\left(D_{S}{ }^{f}\left(x_{0}\right)\right)={D_{S}}^{f}\left(x_{0}\right)$. Now the result follows from theorem 1.14 with $M=D_{S}{ }^{f}\left(x_{0}\right)$. $\square$.

Theorem 2.6: Let $X$ be a $p$-normed space, $f$ and $T$ self-maps of $X$ with $x_{0} \in X$, and $S \in C_{0}$ with $T\left(S_{x_{0}}\right) \subseteq f(S)=S$. Suppose that $\left\|T x-x_{0}\right\|_{p} \leq\left\|x-x_{0}\right\|_{p}$ for all $x \in s_{x_{0}}$, $\left\|f x-x_{0}\right\|_{p}=\left\|x-x_{0}\right\|_{p}$ for all $x \in S$ is compact. Then $P_{S}\left(x_{0}\right)$ is nonempty, closed, and convex and 
$\overline{T\left(P_{S}\left(x_{0}\right)\right)} \subseteq f\left(P_{S}\left(x_{0}\right)\right) \subseteq P_{S}\left(x_{0}\right)$. If, in addition, $f$ and $T$ are continuous and ultra-occasionally weakly compatible on $P_{S}\left(x_{0}\right)$, and $\|T x-T y\|_{p} \leq \psi_{f, T}(x, y)$ for all $x, y \in P_{S}\left(x_{0}\right)$, Then $P_{S}\left(x_{o}\right) \cap F(f, T)$ is nonempty.

Proof : We may assume that $x_{0} \notin S$. If $x \in S \backslash S_{x_{0}}$, then $\|x\|_{p}>2\left\|x_{0}\right\|_{p}$ and, so

$$
\left\|x-x_{0}\right\|_{p} \geq\|x\|_{p}-\left\|x_{0}\right\|_{p}>\|x\|_{p} \geq d_{p}\left(x_{0}, S\right) .
$$

Thus $\alpha=d_{p}\left(x_{0}, S_{x_{0}}\right)=d_{p}\left(x_{0}, S\right)$. Suppose $\overline{T\left(S_{x_{0}}\right)}$ is compact. Since the norm is continuous, there exists $z \in \overline{T\left(S_{x_{0}}\right)}$ such that $\beta=d_{p}\left(x_{0}, \overline{T\left(S_{x_{0}}\right)}\right)=\left\|z-x_{0}\right\|_{p}$. Hence

$$
\alpha \leq \beta \leq d_{p}\left(x_{0}, T\left(S_{x_{0}}\right)\right) \leq\left\|T y-x_{0}\right\|_{p}=\left\|y-x_{0}\right\|_{p}
$$

For all $y \in S_{x_{0}}$. This show $\alpha=\beta$. Thus, $P_{S}\left(x_{0}\right)$ is nonempty, closed, and convex. Note that the compactness of $\overline{T\left(P_{S}\left(x_{0}\right)\right)}$ implies that $T$ is semi compact on $P_{S}\left(x_{0}\right)$. Since $\left\|f x-x_{0}\right\|_{p}=\left\|x-x_{0}\right\|_{p}$

For all $x \in S$ and $\left\|T x-x_{0}\right\|_{p} \leq\left\|x-x_{0}\right\|_{p}$ for all $x \in P_{S}\left(x_{0}\right)$, then $f\left(P_{S}\left(x_{0}\right)\right) \subseteq P_{S}\left(x_{0}\right)$,

$C_{S}{ }^{f}\left(x_{0}\right)=P_{S}\left(x_{0}\right)$, and $\overline{T\left(P_{S}\left(x_{0}\right)\right)} \subseteq P_{S}\left(x_{o}\right)$. Moreover, as $P_{S}\left(x_{0}\right) \subseteq S=f(S)$ and $\left\|f x-x_{0}\right\|_{p}=$ $\left\|x-x_{0}\right\|_{p}$ for all $x \in S$, we have $P_{S}\left(x_{0}\right) \subseteq f\left(P_{S}\left(x_{0}\right)\right)$. Therefore $T\left(P_{S}\left(x_{0}\right)\right) \subseteq f\left(P_{S}\left(x_{0}\right)\right)=P_{S}\left(x_{o}\right)$. The rest follows from theorem 2.7 with $M=P_{S}\left(x_{o}\right)$.

\section{References}

[1] M.A. AL-Thagafi, J. Approx. Theory, 85, pp-318(1996).

[2] M.A. Al-Thagafi, Naseer Shahzad, Acta Math. Sinica 24, pp-867 (2008).

[3] M.A. Al-Thagafi, N. Shahzad, Nonlinear Anal. 64, pp-2778 (2006).

[4] N.Hussain, A.R Khan, Dem. Math., vol. XXXVI (3), pp- 675 (2003).

[5] N.Hussain, M.A. Kutbi, Inter, J. pure, Appl Math., vol. 56 , pp- 487(2009).

[6] G.Kothe, Topological vector spaces.1, springer-Verlag New York Inc., New York, (1969).

[7] J. Kyukim, M. Abas, Bull. Korean Math. Soc. 44, pp-537 (2007).

[8] A.Latif, Arch. Math., 37, pp-71(2001).

[9] H. Shojaei, K. Banaei, N. Shojaei, , J. Math. Computer Sci., 6, pp- 118(2013).

[10] W. Rudin, Functional Analysis, McGraw-Hill, New York, (1973). 